considerable efforts are being made to find better anticancer drugs and it is inevitable that a book of this kind cannot deal with or evaluate all the latest seemingly remarkable agents. It is thus only able to deal cursorily with asparaginase and eytosine arabinoside, in both of which there is currently much interest.

In giving their readers the basis of drug development the authors pinpoint the weakness of experimental cancer chemotherapy: the lack of a reliable predictive screening test for anticancer compounds. They point out that the clinician is only too well aware of the limitations of the two dozen or so drugs he has available for the treatment of neoplastic disease and looks to the organic chemist to provide better ones. Some of the most effective anticancer agents in clinical use today are, however, not so much the result of synthetic organic revelations, but of acute biological and clinical observations.

The desire of the medicinal chemist to see activity related to structure is very understandable, but fraught with obvious and well recognized though rarely heeded hazards. Structure-activity "relationships" are often of a transitory nature and only hold because insufficient compounds were made. Thus the authors state that among alkylating agents it has been established quite unequivocally that only bifunctional compounds achieve cytotoxic effects. Recent reports, however, indicate that there may be monofunctional alkylating agents which have greater antitumour activity than any alkylating agent previously tosted.

The book should be required reading for clinicians and senior medical students, but it would be helpful for research workers if references were given in the toxt instcad of a reading list at the end of the book. It is to be hoped that this can be remedied in future editions.

\section{K. Hellmann}

\section{RADIATION DOSIMETRY}

\section{Thermoluminescent Dosimetry}

By J. R. Cameron, N. Suntharalingam and G. N. Kenney. Pp. xxiii + 232. (University of Wisconsin Press: Madison and London, February 1969.) \$7.50; 71s $6 d$.

THIs book has the groat merit that it is eminently readable and gives an account of the many basic experiments that the authors and others hare carried out. It also provides a good account of the many applications of thermoluminescent dosimetry, demonstrating the versatility of this method compared with many other systems.

The order of presentation of the book is uneonventional in that it presents applications of the method at the beginning before the practice, and theory is left until the last chapter. It is in this respect that whilst the revicwer can read the book drawing on his previous knowledge of the subject, the student would benefit if there had been, early on in the book, a comprehensive summary of the basic procedures and problems that have to be overcome.

The inclusion of the basic design of a reader that a technician could make up in a moderately equipped workshop is good, and so also is the extensive bibliography which includes the titles of the pap ers.

The description of the response of thermoluminescent materials as a lincar response always leads to some confusion in that the response referred to is that obtained when the light ernitted is plotted against the dose as a log-log curve. When the response is plotted on linear scales a linear curve is obviously not in general obtained. It would have been useful to comment on this because many initial experimenters have been confused by this description.

Altogether, this is a very useful book which has been well proofed and I recommend it as an up to date report of the practice of thermoluminescent dosimetry.

R. E. ElLIS

\section{CHEMICAL REAGENTS}

\section{Complexometry with EDTA and Related Reagents}

By T. S. West. Third edition. Pp. 235. (BDH Chemicals: Poole, 1969.) 50s.

THE monograph is a third edition, completely revised and rewritten, and gives a thorough coverage of the literature up to 1964 and an occasional paper in 1965. This suggests that the work with ethylenediaminetetra-acetic acid (EDTA) and related reagents has completed its present phase of thorough investigation. Hence the monograph provides a most useful and lasting survey of the field, which has been extensively developed since 1945, following Schwarzenbach's use of cthylenediaminetetra-acetic acid. This reagent gives an almost instantaneous formation of stable, water-soluble chelate complexes with most metal ions in solution.

The survey is in a most useful summary form and is arranged in eight sections. An introduction gives the theory underlying the formation of complexes, the essential properties of a complexometric reagent, and end-point detection, including instrumental procedures. The emphasis, however, is on the reliability of visual indicator's in view of their ease of application and ready automation by photoelectric methods. The section on EDTA concisely reports the reactions, stoichiometry and the relationship between complex stability, $p \mathrm{H}$ and temperature. $\mathrm{A}$ series of tables gives the logarithms of the stability constants for the various classes of complexones.

The many indicators employed fall into distinct classes and with concise reports on the main metallochromic, fluorescent, redox and chemiluminescent types. Each indicator is thoroughly covered in a distinctly attractive summary form, with full reference cover to the original papers. Then follow the many physico-chemical methods of end-point detection. Again the conciseness and clarity of the descriptions for conductometric, high-frequency, potentiometric, amperometric, spectrophotometric, chronopotentiometric, enthalpimetric, ete.. titrations are noteworthy. The use of masking agents considerably extends the analytical scope.

Although many other compounds varying in one way or another the structure of complexometric titrants have been examined, the modifications have not approached EDTA in usefulness, though for each modification the essential characteristics are given. The presentation is a model of effective condensation of very many publications giving precisely the particular direction in which the titrants. may be useful.

The last section covers selected EDTA procedures for some fifty-seven elements, outlining the reagents and method for the use of most of these. The monograph is, however, in no sense a laboratory manual though sufficient information is given for any necessary determinations. Of special note is the use of the method on water analysis using visual end-points.

The publication is associated with British Drug Houses, $\mathrm{Ltd}$, and many of the indicators are commercially availablo, but not the range of titrants. The index to the book usefully gives the full names of titrants often met only as abbreviations. Although there is a full bibliography, a separate author index is not included.

The monograph is marred by many errors linked with the structures given for indicators. These range from incorrect bonding for an aromatic nucleus on pages 48 , 56 and 66. Formulations showing carbonium ions, as for lucigenin, would more usually utilize anthracene type bonding with charges on nitrogen. The charge on carbon is also shown for catechol violet, though on the facing page the elosely related pyrogallol red is given the usual fluoresccin-type structure. Similarly, methyl thymol blue and glycine thymol blue are more usually shown with a $p$-quinonoid ring, and this applies also for xylenol orange and semi-xylenol orange. 'Then morin is a $2^{\prime}: 4^{\prime}$ substituted anthocyanidin and not $2^{\prime}: 5^{\prime}$ as given, and 